\title{
Energy dependence of the freeze out eccentricity from the azimuthal dependence of HBT at STAR
}

\author{
Christopher Anson (for the STAR Collaboration) \\ Department of Physics, The Ohio State University, Columbus, OH 43210, USA \\ E-mail: anson.9@osu.edu
}

\begin{abstract}
.
Non-central heavy ion collisions create an out-of-plane-extended participant zone that expands toward a more round state as the system evolves. The recent RHIC Beam Energy Scan at $\sqrt{s_{N N}}$ of $7.7,11.5$, and $39 \mathrm{GeV}$ provide an opportunity to explore the energy dependence of the freeze out eccentricity. The new low energy data from STAR complements high statistics data sets at $\sqrt{s_{N N}}$ of 62.4 and $200 \mathrm{GeV}$. HanburyBrown-Twiss (HBT) interferometry allows to determine the size of pion emitting source regions. The dependence of the HBT radius parameters on azimuthal angle relative to the reaction plane have been extracted. These dependencies can be related to the freeze out eccentricity. The new results from STAR are consistent with a monotonically decreasing freeze out eccentricity and constrain any minimum, suggested by previously available data, to lie in the range between 11.5 and $39 \mathrm{GeV}$. Of several models UrQMD appears to best predict the STAR and AGS data.
\end{abstract}

\section{Introduction}

One of the primary goals of heavy ion physics is to explore the phase diagram of QCD matter by mapping out the nature of the transition between a deconfined and hadronic state. The recent Beam Energy Scan at RHIC allows to search for signs of a change in the Equation of State of hot, dense QCD matter produced in heavy ion collisions. One of the main observables that is sensitive to the Equation of State is the freeze-out shape of the participant zone in non-central collisions.

At its creation, the participant zone forms an out-of-plane extended ellipsoid, with an initial eccentricity (in the transverse plane) in non-central heavy ion collisions. The material, being more compressed in the reaction plane, experiences larger inplane pressure gradients compared to the out-of plane direction. Preferential in-plane expansion drives the participant zone toward a more circular freeze out shape. Systems with a longer lifetime, larger in-plane versus out-of-plane pressure gradients, or both, achieve a more round shape or could even concievably become in-plane extended. Based solely on these two considerations, we would expect the excitation function for the freeze out eccentricity to fall monotonically with increasing energy. 
However, the five measurements available prior to the Beam Energy Scan at 2.7, 3.32, and $3.84 \mathrm{GeV} \mathrm{[1],} \mathrm{at} 17.3 \mathrm{GeV}$ [2], and at $200 \mathrm{GeV} \mathrm{[3],} \mathrm{suggested} \mathrm{a} \mathrm{possible}$ minimum followed by a rise in the excitation function with increasing energy. It has been speculated [4] that such a scenario may somehow be explained by a softening of the equation of state due to entrance, above some energy, into a mixed phase. As the pressure gradients vanish in a mixed phase, the shape would not change during this portion of the lifetime. This may be observed as a plateau or perhaps even a minimum in the excitation function.

The suggestion of possible non-monotonic behaviour and the availability of BES data provide motivation and opportunity for extracting the freeze out shape. The means is provided by HBT analysis relative to the event plane.

\section{Method}

HBT analyses provide information about the shape of the particle emitting source regions in heavy ion collisions expressed in terms of radii in the Bertsch-Pratt (out,side,long or o,s,l) coordinate system. In the current analysis, HBT radii are measured relative to the $2^{\text {nd }}$ order event plane determined using charged particles in the STAR Time Projection Chamber. The correlation functions are constructed using

like-signed pion pairs with average transverse pair momenta, $k_{T}=\left(p_{T 1}+p_{T 2}\right) / 2$, in the range $0.15<k_{T}<0.60 \mathrm{GeV} / \mathrm{c}$. After corrections, discussed below, are applied to both the same event and mixed event pair distributions the radii are extracted by fitting a correlation function of the form

$$
C(\mathbf{q})=N\left\{(1-\lambda)+\lambda K_{c}\left[1+\exp \left(-q_{o}^{2} R_{o}^{2}-q_{s}^{2} R_{s}^{2}-q_{l}^{2} R_{l}^{2}-2 q_{o} q_{s} R_{o s}^{2}\right)\right]\right\}(1)
$$

using a fit range of $\left|q_{i}\right|<0.15 \mathrm{GeV} / \mathrm{c}, i=o, s, l$. $\mathrm{N}$ is a normalization factor, $\lambda$ the fraction of pairs that interact, $R_{i}$ the HBT radii in the out-side-long coordinate system, $q_{i}$ the relative momenta, $\mathbf{q}=\mathbf{p}_{\mathbf{1}}-\mathbf{p}_{\mathbf{2}}$, projected on the out-side-long coordinate system, and $K_{c}$ the Coulomb interaction for each $q_{o}, q_{s}, q_{l}$ bin. The extracted radii exhibit $2^{\text {nd }}$ order oscillations relative to the event plane expressed using Fourier coefficients as

$$
\begin{array}{ll}
R_{i}^{2}(\phi)=R_{i, 0}^{2}+2 \Sigma_{n=2,4,6, \ldots} R_{i, n}^{2} \cos (n \phi) & i=o, s, l \\
R_{i}^{2}(\phi)=2 \Sigma_{n=2,4,6, \ldots} R_{i, n}^{2} \sin (n \phi) & i=o s
\end{array}
$$

where $0<\phi<\pi$ is the angle from the event plane at which the radii are observed.

The freeze out eccentricity can be approximated [5, 6] using

$$
\varepsilon_{F}=\frac{\sigma_{y}^{2}-\sigma_{x}^{2}}{\sigma_{y}^{2}+\sigma_{x}^{2}} \approx 2 \frac{R_{s, 2}^{2}}{R_{s, 0}^{2}},
$$

where $\sigma_{x}^{2}$ and $\sigma_{y}^{2}$ are the in- and out-of-plane length scales. More in depth discussion of this method of analysis can be found in [5].

The amplitude of the oscillation plays a direct role in the calculation and extracting the correct value requires correction for two effects: finite reaction plane resolution, and 


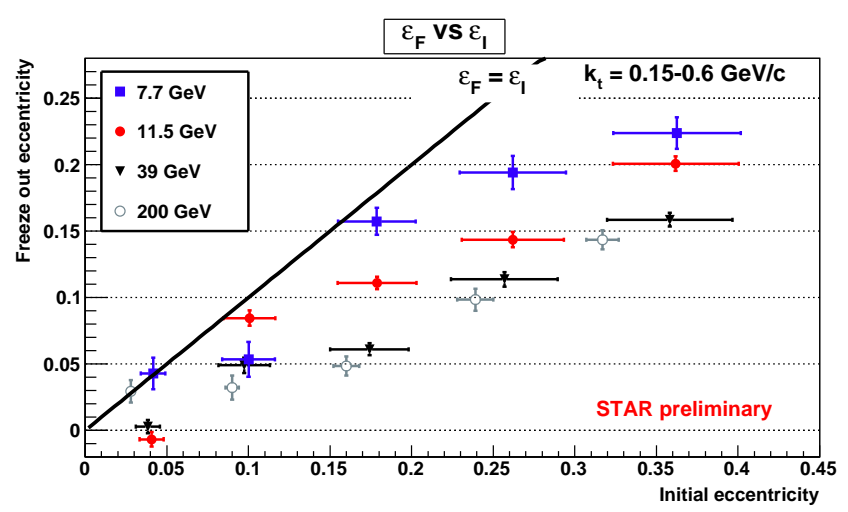

Figure 1. $\varepsilon_{F}$ versus $\varepsilon_{I}$. Points further below the line $\varepsilon_{F}=\varepsilon_{I}$ change shape more than points closer to the line. Errors are statistical only. Color online.

finite bin width. Uncertainty in the computed reaction plane mixes pairs in adjacent bins, reducing the extracted oscillation amplitude. Similarly, pairs within a finite bin width correspond not to single source region but to a range of source regions with a range of sizes. The extracted radii in a bin are averages which again reduces the observed oscillation. A model independent correction algorithm [7] compensates for both effects.

\section{Results}

The shape evolution for different energies is demonstrated in figure 1. For all energies the freeze out eccentricity generally increases from central to peripheral collisions. The shape changes relatively little for the central collisions because of the smaller difference between in- and out-of-plane pressure gradients. In more peripheral collisions the shape changes more but in all cases the evolution is such that the shape tends to become more circular while remaining out-of-plane extended. The amount of change in shape, at least for the $10-80 \%$ centrality range, shows an energy dependence. The $7.7 \mathrm{GeV}$ results lie closest to the line $\varepsilon_{F}=\varepsilon_{I}$ which means the shape has changed the least. At $11.5 \mathrm{GeV}$ the system reaches a noticeably more round shape and from 11.5 to $39 \mathrm{GeV}$ there is again a noticeable change. In a much wider range from $39 \mathrm{GeV}$ to $200 \mathrm{GeV}$ the shape continues to evolve a similar amount, relatively independent of collision energy.

The excitation function, figure 2, is constructed by averaging the results in the $10-20 \%$ and $20-30 \%$ centrality range to obtain a result comparable to results from other experiments. The errors include statistical errors as well as preliminary systematic errors from track and pair cuts; further sources of systematic error being investigated but each is expected to be small. Taken alone, the STAR data are consistent with a monotonically decreasing shape. Any minimum, if confirmed, is constrained to the region between $11.5 \mathrm{GeV}$ and $39 \mathrm{GeV}$. If a minimum is not confirmed changes in the slope of the excitation function might still be related to changes in the equation of state through detailed model comparisons.

Comparison to models, from [4, shows that the prediction from UrQMD comes 


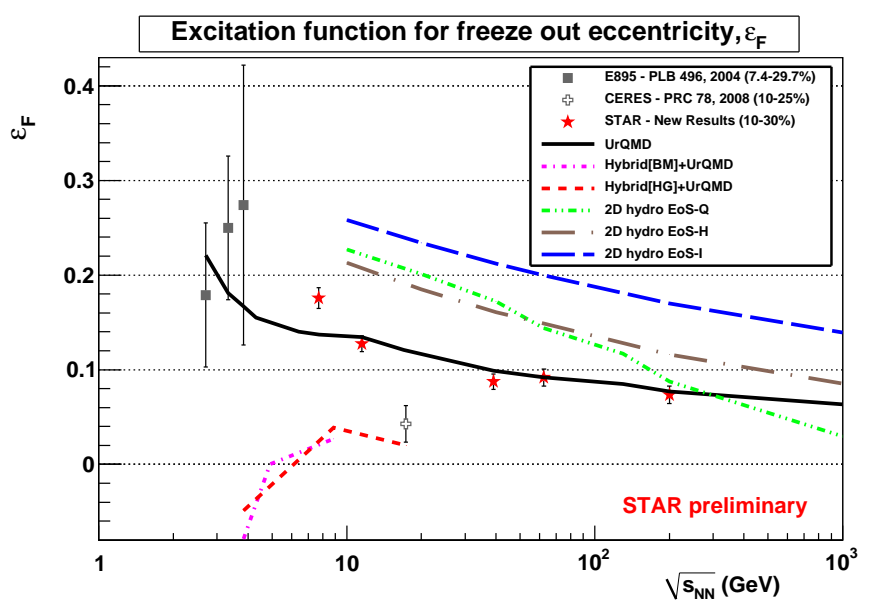

Figure 2. Freeze out eccentricity, $\varepsilon_{F}$, as a function of $\sqrt{s_{N N}}$ for data and models [4] with similar centralities. Color online.

closest to describing the results from STAR and AGS. The hybrid models tend to come close to the CERES point but underpredict the rest while the 2D hydrodynamic predictions tend to overpredict the data at most energies. An interesting comparison between 2D hydrodynamic models with hadronic (EoS-H) and ideal quark gas (EoS-I) equations of state shows the sensitivity of the freeze out shape to changes in the equation of state so the new data provides an important opportunity to further constrain models.

\section{Summary}

Energy dependence of the freeze out eccentricity suggest the amount of change in shape from the initial value increases relatively rapidly at lower energies but less rapidly at higher energies. If a minimum is confirmed it is constrained to lie between 11.5 and $39 \mathrm{GeV}$. Recently obtained $19.6 \mathrm{GeV}$ and $27 \mathrm{GeV}$ data will allow to study the excitation function in this interesting region with the same detector, acceptance and analysis techniques. Detailed comparisons will further constrain models, contributing to a more accurate description of heavy ion collisions over a range of energies that access much of the phase diagram of hot QCD matter.

\section{References}

[1] Lisa M et al 2000 Phys. Lett. B 496 1-8

[2] Adamova D et al 2008 Phys. Rev. C 78, 064901

[3] Adams J et al 2005 Phys. Rev. C 7144906

[4] Lisa M A, Frodermann E, Graef G, Mitrovski M, Mount E, Petersen H and Bleicher M 2011 New J. Phys. 13065006

[5] Mount E, Graef G, Mitrovski M, Bleicher M and Lisa M A 2010 arXiv:1012.5941

[6] Retiere F and Lisa M A 2004 Phys. Rev. C 70044907

[7] Heinz U W, Hummel A, Lisa M A and Wiedemann U A 2002 Phys. Rev. C 66044903 